\title{
Electron structure and electron-phonon interaction in the strongly correlated electron system of cuprates
}

\author{
S.G. Ovchinnikov, V.A. Gavrichkov, M.M. Korshunov, and E.I. Shneyder \\ L.V. Kirensky Institute of Physics, Siberian Branch of Russian Academy of Sciences \\ Krasnoyarsk 660036, Russia \\ E-mail: mkor@iph.krasn.ru
}

Received August 22, 2005

\begin{abstract}
The generalized tight-binding method presents a practical realization of the scheme that describes quasiparticles in strongly correlated electron system and consists of exact intra-cell diagonalization of the model Hamiltonian and perturbative treatment of the inter-cell hoppings. In present paper this method and its ab initio modification applied to undoped and weakly doped HTSC cuprates. Results are in very good agreement with the experimental ARPES data on various compounds. Starting with multiband $p-d$ model the realistic effective low-energy Hamiltonian of strongly correlated electrons interacting with spin fluctuations and phonons is derived both for hole and electron doped systems. Without electron - phonon interaction the pure magnetic mechanism of pairing does not provide the correct value of $T_{c}$ even for single-layer $\mathrm{La}_{2-x} \mathrm{Sr}_{x} \mathrm{CuO}_{4}$ and $\mathrm{Nd}_{2-x} \mathrm{Ce}_{x} \mathrm{CuO}_{4}$.
\end{abstract}

PASC: 74.72.-h; 74.20.Mn; 74.25.Jb; 74.25.Kc

Keywords: strongly correlated electron system, elektron-phonon interaction HTSP cuprates.

\section{Introduction}

The last 20 years after the discovery of superconductivity in cuprates [1] have been a time of intensive theoretical and experimental studies of these and related materials. The rich phase diagram of doped cuprates proves that physics of cuprates is much more complicated that of conventional metallic superconductors. Now it is clear that it is strong electron correlations (SEC) that result in many unusual properties both of the normal and superconducting phases. Due to SEC a conventional band theory fails to give a description of cuprates in a wide doping region, especially in the underdoped-optimally doped interval. Without a reliable $a b$ initio method the theory has to involve a model approach, and a large variety of models with different mechanisms of pairing have been proposed. That is why the study of the electronic properties in the undoped cuprates and its evolution with doping is so important: it gives the basis for more reliable description of the superconducting state and mechanism of superconductivity. There are several reviews in the literature on the similar subject [2-6]; a lot of new interesting results appear recently that will be considered in this paper. We want to discuss not only the achievements, but also the unsolved problems in this field.

The paper is organized as follows: the Sec. 2 contains a description of the generalized tight-binding (GTB) method developed for SEC systems [7-9]. The band structure and comparison to ARPES data is discussed in the Sec. 3. Section 4 contains the effective electronic Hamiltonians for $n$ - and $p$-type doped cuprates and the magnetic mechanism of pairing. Section 5 is devoted to the electron - phonon interaction in systems with SEC. The conclusion and a discussion of some future perspectives are given in Sec. 6 .

\section{The generalized tight binding method for strongly correlated electron systems}

The failure of conventional single electron approach in systems with SEC makes it necessary to reconsider what the renormalized electron is when the interaction between electrons is much larger then its kinetic energy. Evidently that conventional free-electron starting point with a perturbative treatment of correlations is not an adequate approach. Very often 
the electron in the SEC limit is discussed as a composite particle: spinon-holon, slave-boson, slave-fermion and other representations are known in the literature (e.g., see [10]). All of these decompositions are made by hand, and the very fact of their existence proves that none of them is true. We will define an electron as a combination of quasiparticles with charge $e$ and spin $1 / 2$ with odd spectral weight. This language is based on the exact Lehmann representation [11].

At $T=0$ there is the exact representation for a single-electron Green function

$$
G_{\sigma}(k, \omega)=\sum_{m}\left(\frac{A_{m}(k, \omega)}{\omega-\Omega_{m}^{+}}+\frac{B_{m}(k, \omega)}{\omega-\Omega_{m}^{-}}\right),
$$

where the electron addition energy $\Omega_{m}^{+}$and electron removal energy $\Omega_{m}^{-}$, given by

$$
\begin{aligned}
& \Omega_{m}^{+}=E_{m}(N+1)-E_{0}(N)-\mu, \\
& \Omega_{m}^{-}=E_{0}(N)-E_{m}(N-1)-\mu,
\end{aligned}
$$

have the same meaning as the quasiparticle (QP) (electron and hole) in the Landau Fermi-liquid theory. The matrix elements

$$
\begin{gathered}
A_{m}(k, \omega)=\left|\left\langle 0, N\left|a_{k \sigma}\right| m, N+1\right\rangle\right|^{2}, \\
B_{m}(k, \omega)=\left|\left\langle m, N-1\left|a_{k \sigma}\right| 0, N\right\rangle\right|^{2},
\end{gathered}
$$

provide the charge $e$ and spin $1 / 2$ for each QP, and the odd spectral weight less then a free-electron spectral weight that is equal to 1 . Here $|m, N\rangle$ denotes the $m$-th many-electron eigenstate of a system with $N$ electrons,

$$
H|m, N\rangle=E_{m}|m, N\rangle \text {. }
$$

Thus we read the Eq. (1) in a following way: the electron is a linear combination of different QP with charge $e$, spin $1 / 2$, and odd spectral weight.

Thus the Lehmann representation gives us the proper language to describe the renormalized electron in SEC system. But it is useless practically, because we do not know the exact eigenstates $|m, N\rangle$ and eigenenergies $E_{m}$, and we cannot calculate the QP energy and its spectral weight. The GTB method is a perturbative realization of this language. The Hubbard $X$-operators [14] appear to be a most adequate mathematical tool for this language.

The GTB method [7-9] consists of 3 steps:

i) the exact diagonalization of the multielectron Hamiltonian inside the unit cell (for cuprates we take a $\mathrm{CuO}_{6}\left(\mathrm{CuO}_{4}\right)$ cluster as unit cell);

ii) construction of the Hubbard operators with the help of cell eigenstates $|p\rangle$ :

$$
X_{f}^{p q} \leftrightarrow X_{f}^{n}
$$

Here we introduce a more simple notation in which each pair $(p, q)$ of the initial $q$ and final $p$ states is denoted by a number $n$;

iii) the intercell hoppings and interactions $\hat{t}(q)$ are considered as a perturbation by the diagram technique for $X$ operators $[15,16]$. The series of perturbation theory are constructed for the matrix Green function,

$$
D_{n n^{\prime}}(k, \omega)=\left\langle\left\langle X_{k}^{n} \mid \begin{array}{c}
+ \\
X_{k}^{n^{\prime}}
\end{array}\right\rangle\right\rangle_{\omega},
$$

rather than for the electron Green function related to the former in the $X$ representation as

$$
G_{\lambda \lambda^{\prime}, \sigma}(k, \omega)=\sum_{n n^{\prime}} \gamma_{\lambda \sigma}(n) \gamma_{\lambda^{\prime} \sigma}^{*}\left(n^{\prime}\right) D_{n n^{\prime}}(k, \omega)
$$

Analysis of a general structure of the Green function (6) revealed the following generalized Dyson Eq. [9]

$$
\hat{D}\left(\mathbf{q}, \omega_{n}\right)=\left\{\left[G^{(0)}\left(\mathbf{q}, \omega_{n}\right)\right]^{-1}+\hat{\Sigma}\left(\mathbf{q}, \omega_{n}\right)\right\}^{-1} \hat{P}\left(\mathbf{q}, \omega_{n}\right),
$$

where $G^{(0)}\left(\mathbf{q}, \oplus_{n}\right)$ is the Hartree-Fock Green function in the $X$-operator diagram technique (that is equal to the Hubbard-I approximation result), and given by

$$
\left[\hat{G}^{(0)}\left(\mathbf{q}, \omega_{n}\right)\right]^{-1}=\left[\hat{G}_{0}\left(\omega_{n}\right)\right]^{-1}-P\left(\mathbf{q}, \omega_{n}\right) \hat{t}(q),
$$

and $\hat{G}_{0}\left(\omega_{n}\right)$ is the local (intracell) propagator. Besides the self-energy $\hat{\Sigma}\left(\mathbf{q}, \omega_{n}\right)$ there is also a strength operator $P\left(\mathbf{q}, \oplus_{n}\right)$, which determines the QP spectral weight (oscillator strength). The strength operator was introduced early in the spin operator diagram technique [17].

The Hartree-Fock approximation is equivalent to the Hubbard-I approximation for the intercell hopping $\hat{t}$. In this approximation the dispersion equation for the QP bands is given by

$$
\operatorname{det}\left\|\delta_{n n^{\prime}}\left(\omega-\Omega_{n}\right) / F(n)-t_{n n^{\prime}}(k)\right\|=0 .
$$

The QP band structure in GTB dependents on a set of microscopic model parameters that should be fitted to some experimental data or calculated separately. The set of parameters of the multiband $p-d$ model proposed by Gaididei and Loktev [18] has been fitted in [8] to ARPES data for $\mathrm{Sr}_{2} \mathrm{CuO}_{2} \mathrm{Cl}_{2}$, and it was fixed to study the doping dependence of the QP band structure [19]. Recently a hybrid LDA+GTB scheme has been developed that allows to find the parameters by the $a b$ initio method [20]. 


\section{Doping-dependent quasiparticle band structure}

We calculate the QP band structure in the framework of the multiband $p-d$ model [18]. The exact diagonalization of the model Hamiltonian for $\mathrm{CuO}_{6}$ cluster results in a set of following terms (in a hole representation) shown in the Fig. 1. Here vacuum state $|0\rangle$ corresponds to the $d^{10} p^{6}$ configuration, single-hole $|\sigma\rangle, \sigma= \pm 1 / 2$, sates are the hole molecular orbitals (mixture of $d^{9} p^{6}$ and $d^{10} p^{5}$ ) with $b_{1 g}$ symmetry. The most important difference of the multiband model compare to the three-band model $[21,22]$ is quite small difference in energies in the 2-hole sector of Hilbert space between the ${ }^{1} A_{1 g}$ singlet (with large contribution of the Zhang-Rice $d^{9} p^{5}$ configuration) and ${ }^{3} B_{1 g}$ triplet. That is why we have to consider both singlet and triplet final states for hole-addition excitation from the $b_{1 g}$ initial state.

The QP band structure obtained by GTB method and corresponding experimental ARPES data are shown for two undoped cuprates: $\mathrm{Sr}_{2} \mathrm{CuO}_{2} \mathrm{Cl}_{2}$ (Fig. 2, $a$ [8]), and $\mathrm{Bi}_{2} \mathrm{Sr}_{2} \mathrm{Ca}_{1-x} \mathrm{Y}_{x} \mathrm{Cu}_{2} \mathrm{O}_{8+y}$ (Fig. 2,b [23]). The dots with vertical bar represent the ARPES data. The bands shown in the Fig. 2 from the top of the valence band separated by the charge-transfer gap, $E_{g} \approx 2 \mathrm{eV}$, from the bottom of the conductivity band. Keeping in mind singlet and triplet final state for forming the top of the valence band fermionic QP one may call these bands «singlet» and «triplet». The hybridization of singlet and triplet bands is nonzero. Usually, the triplet band contribute well below the top of the valence

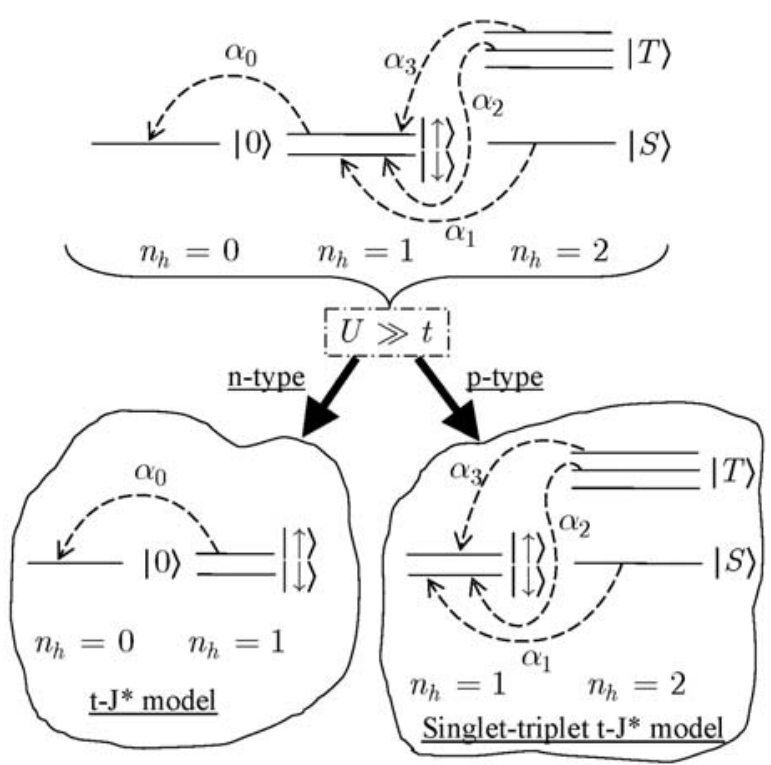

Fig. 1. A scheme of the local multihole eigenstates of the multiband $p-d$ model. Only lowest in energy terms for $n_{h}=1$ and $n_{h}=2$ are shown. Curved dashed lines with arrows indicate different quasiparticle excitations.
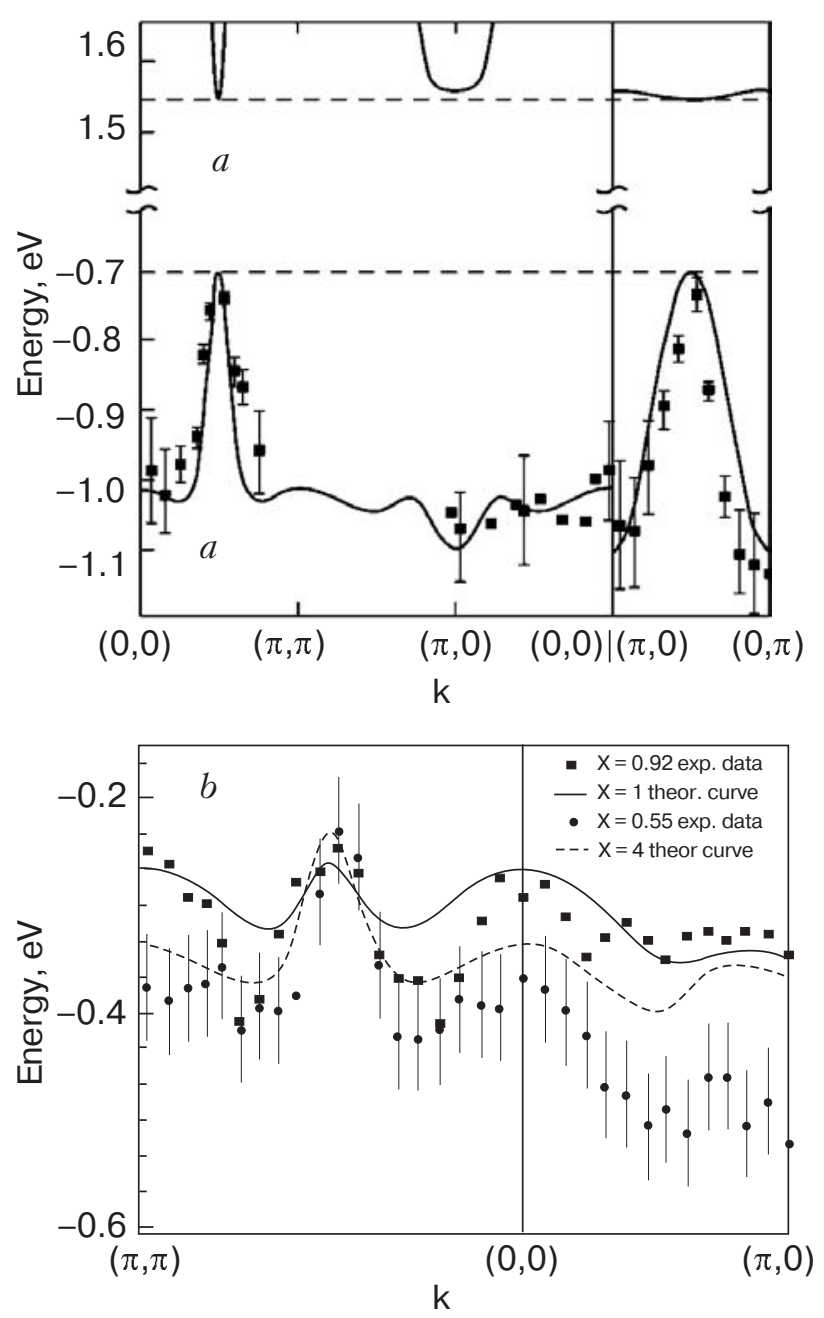

Fig. 2. The quasiparticle dispersion for the top of the valence band in $\mathrm{Sr}_{2} \mathrm{CuO}_{2} \mathrm{Cl}_{2}$ [8] (a) and in $\mathrm{Bi}_{2} \mathrm{Sr}_{2} \mathrm{Ca}_{1-x} \mathrm{Y}_{x} \mathrm{Cu}_{2} \mathrm{O}_{8+y}$ [23] (b) and its comparison to the ARPES data.

band. Nevertheless, in the Fig. 2,b the maxima at $(0,0)$ and $(\pi, \pi)$ points at high $Y$ concentration are related to the triplet band. The anisotropic chemical pressure results in increasing $\mathrm{Cu}-\mathrm{in}$-plane $\mathrm{O}$ distance and decreasing $\mathrm{Cu}-$ apical $\mathrm{O}$ distance and corresponding changes of the oxygen $p-p$ hopping [23].

Situated just above the top of the valence band is the dispersionless band, which is shown by the dotted line in Fig. 2,a. We call it the «virtual» band [8]; this band cannot appear in the conventional single electron approach. Its spectral weight and dispersion are proportional to the doping $x$ and the concentration of magnons [24]. Therefore, for undoped compounds, in the Hubbard-I approximation used in GTB method, the «virtual» band is formed by excitation from the empty (at $T=0$ ) spin minority single hole orbital $b_{1 g}$ to the empty (for doping $x=0$ ) two-hole singlet ${ }^{1} A_{1 g}$, and thus its spectral weight is zero. With hole doping in $\mathrm{La}_{2-x} \mathrm{Sr}_{x} \mathrm{CuO}_{4}$ the ${ }^{1} A_{1 g}$ singlet becomes occupied with probability $x$, and the virtual band transforms into the in-gap band with spectral weight proportional 


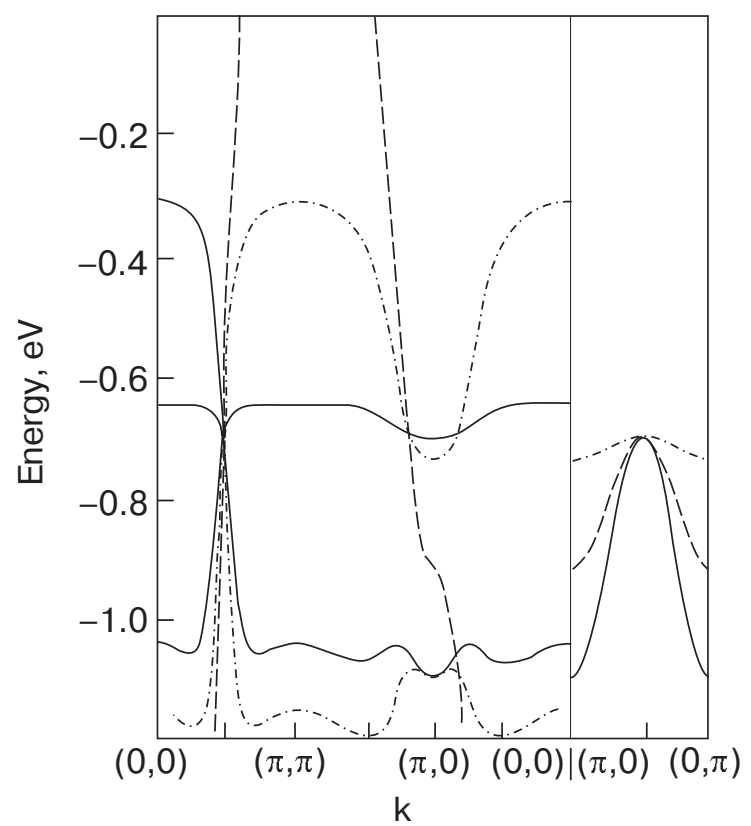

Fig. 3. The doping dependence of the quasiparticle band structure [8].

to $x$ (Fig. 3, [8]). We emphasize that it is not an impurity band, because there is no disorder in the model. All parameter of the Hamiltonian are fixed the same as at $x=0$, and only the hole concentration $n_{h}=1+x$ varies with doping. Of course, this is a rather simplified approach that requires further investigation to understand the evolution of the band structure and the Fermi surface with doping. While the doping dependence of the chemical potential calculated from the GTB band structure [25] is in good agreement with the experimental data [26], the evolution of the Fermi surface with doping does not agree with the ARPES data. We suppose three factors may be important: 1) the dynamical self-energy contribution that exists in GTB beyond the Hubbard-I approximation, 2) an electron-phonon interaction also results in the self-energy renormalization of the QP band structure and the Fermi surface in the normal phase, 3) an impurity scattering of the QP due to compositional disorder induced by $\mathrm{La} / \mathrm{Sr}$ substitution.

\section{The effective low energy Hamiltonian for magnetic mechanism of pairing}

In the GTB method for undoped cuprates we have the filled valence band, the empty conduction band, and the interband hopping. Similar to the procedure of derivation of the $t-J$ model from the Hubbard model, we may exclude the interband hopping by the unitary transformation. It appears that the effective low energy Hamiltonians are different for the holeand electron-doped cuprates [27]. The projected basis of the unit cell for $n$-type doping is similar to the $t-J$ model basis: vacuum state $|0\rangle$ and a single-hole state $|\sigma\rangle, \sigma=\uparrow, \downarrow$ (see Fig. 1). That is why the effective Hamiltonian for electron-doped cuprates is given by the $t-t^{\prime}-t^{\prime \prime}-J^{*}$ model [28]. Here the star denotes that the three-site correlated hopping $H_{3}$ is included. This term is proportional to $J$ and usually omitted in the derivation of the $t-J$ model. As will be discussed below, this term strongly suppress the $T_{c}$ in the $t-J^{*}$ model [29].

For the hole-doped cuprates the projected basis is more complicated in comparison to the $t-J$ model (Fig. 1) and includes single-hole $|\sigma\rangle, \sigma=\uparrow, \downarrow$, singlet $|S\rangle$, and triplet $|T, M\rangle, \quad M=+1,0,-1$ local states. That is why exclusion of the interband hopping results in the two-band effective model, called in [27] the singlet-triplet $t-J$ model. The Hamiltonian of the singlet-triplet $t-t^{\prime}-t^{\prime \prime}-J^{*}$ model is investigated in [30].

A magnetic mechanism of superconductivity with $d$-symmetry in the framework of the $t-J$ model is well known [31,32]. Due to the three-site correlated hoppings several additional diagrams for the anomalous self-energy part appear [29]. Their main effect is a renormalization of the coupling constant in the equation for $T_{c}$ :

$$
1=\frac{n \cdot J / 2}{N} \sum_{q} \frac{\left(\cos q_{x}-\cos q_{y}\right)^{2}}{\tilde{\varepsilon}_{q}-\mu} \tanh \frac{\tilde{\varepsilon}_{q}-\mu}{2 T_{c}},
$$
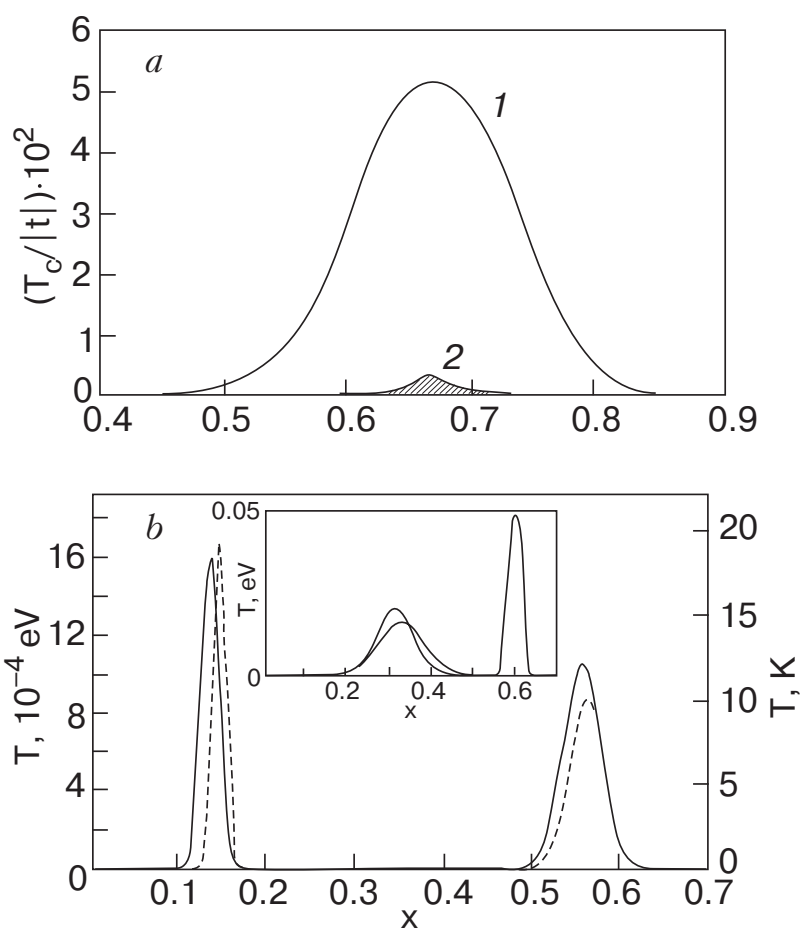

Fig. 4. Critical superconductive transition temperature in the magnetic mechanism of pairing, calculated in the $t-J^{*}$ model [28] $(a)$, and in the singlet-triplet $t-t^{\prime}-t^{\prime \prime}-J^{*}$ model [29] (b). 
where $\tilde{\varepsilon}_{q}$ is a quasiparticle energy in the normal state, also renormalized by three-site interactions [29]. The coupling parameter $J$ of the $t-J$ model is replaced by a parameter $n \cdot J / 2<J / 2$. The suppression of $T_{c}$ by the three-site correlated hopping is shown in Fig. 4, $a$. Instead of $100 \mathrm{~K}$ at the optimal doping in the $t-J$ model we have $4 \mathrm{~K}$ in the $t-J^{*}$ model due to the effect of $\mathrm{H}_{3}$.

A similar calculation of the $T_{c}$ for hole-doped cuprates in the framework of the singlet-triplet $t-t^{\prime}-t^{\prime \prime}-J^{*}$ model with model parameters obtained ab initio [20] also can reproduce up to $50 \%$ of $T_{c}$ in $\mathrm{La}_{2-x} \mathrm{Sr}_{x} \mathrm{CuO}_{4}$ [30]. Possible higher order corrections usually only decrease $T_{c}$ of the mean-field-type approximation. Thus we conclude that pure spin-fluctuation mechanism of pairing is not enough to get the experimental value of $T_{c}$, and some other mechanisms must be involved.

\section{Electron-phonon interactions in strongly correlated cuprates}

Despite the fact that strong correlations and $d$-wave mediated pairing suggest the importance of a spin-based pairing interaction, a number of experiments, such as IR and Raman spectroscopy, neutron scattering, transport, tunneling, ARPES, etc., give convincing evidence the electron - phonon interaction (EPI) is sufficiently strong in HTSC oxides and contributes to pairing (see special issue Phys. Status Solidi 242, No. 1 (2005)). With the purpose of further development of the theory we should take into account both spin fluctuations and EPI in regime of strong electron correlations.

Having this in mind, we describe EPI in cuprates starting from a realistic multiband $p-d$ model. Changes of Hamiltonian parameters due to atomic displacements are calculated to linear order in the displacements. In the framework of the GTB method we obtain

$$
\begin{gathered}
H_{\mathrm{el}-\mathrm{ph}}=\sum_{\mathbf{k q} v} \sum_{m m^{\prime}} g_{m m^{\prime}}^{(v)}(\mathbf{k}, \mathbf{q})\left(X_{\mathbf{k}+\mathbf{q}}^{m}\right)^{+} X_{\mathbf{k}}^{m^{\prime}}\left(b_{\mathbf{q}, v}+b_{-\mathbf{q}, v}^{+}\right) \\
g_{m m^{\prime}}^{(v)}(\mathbf{k q})=\delta_{m m^{\prime}}^{(v)} g_{\mathrm{dia}, m}^{(\mathbf{q})}(\mathbf{q})+g_{\mathrm{off}, m m^{\prime}}^{(v)}(\mathbf{k}, \mathbf{q})
\end{gathered}
$$

where the Hubbard operator $X_{k}^{m}$ annihilates a hole with quasiparticle index $m$ and wave vector $\mathbf{k}$, and operator $b_{\mathbf{q}, v}^{+}$creates a phonon with wave vector $\mathbf{q}$, mode index $v$, and frequency $\omega_{v}(\mathbf{q})$. The total matrix element of the EPI is presented as the sum of a diagonal part $g_{\mathrm{dia}, m}^{(v)}(\mathbf{q})$ depending on $\mathbf{q}$ (scattering momentum of the electron), and an off-diagonal part $g_{\mathrm{off}, m m^{\prime}}^{(v)}(\mathbf{k}, \mathbf{q})$ depending also on $\mathbf{k}$ (initial momentum, see details in [33]).
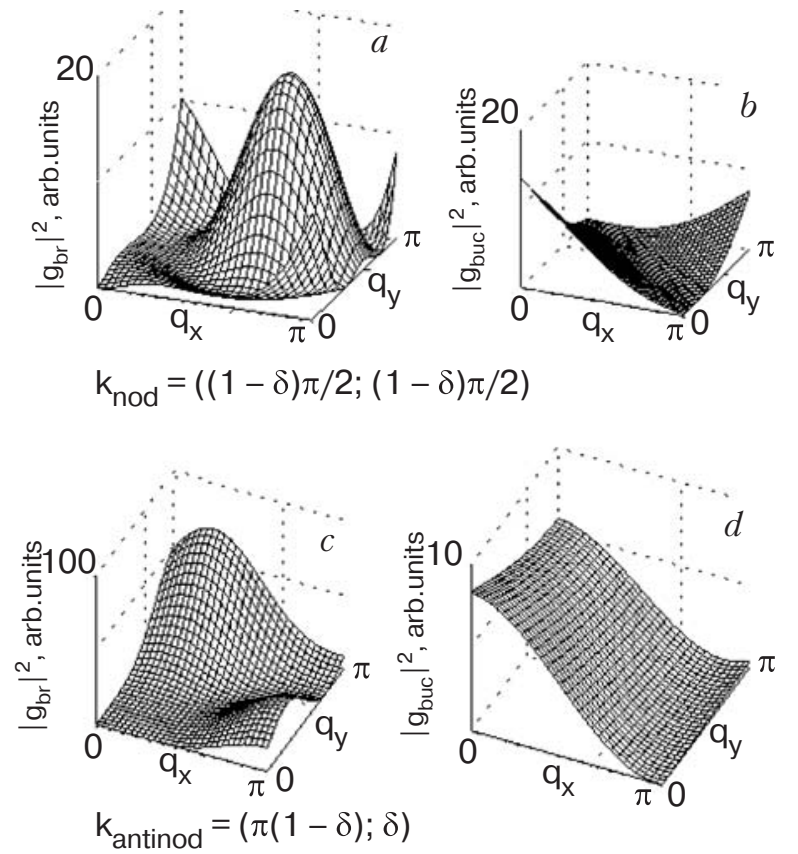

Fig. 5. Plots of the EPI coupling for breathing $\left(\left|g_{\mathrm{br}}\right|^{2}\right)$ and buckling $\left(\left|g_{\text {buc }}\right|^{2}\right)$ modes as a function of scattered momentum $q$ for initial state in the nodal $(a),(b)$ and antinodal $(c),(d)$ points.

We restrict consideration to three phonon modes which are most strongly coupled to electrons [34], i.e., the vibration of the in-plane oxygen along $\mathrm{Cu}-\mathrm{O}$ plane and along the $z$-axis (breathing mode and buckling mode, correspondingly), and the vibration of the apex oxygen in the $z$ direction (apex breathing mode). Explicit dependence of matrix elements on $\mathbf{k}$ and $\mathbf{q}$ was obtained taking into consideration the symmetry of atomic displacements for each mode. Plots of the EPI coupling for the breathing and buckling modes are shown in Fig. 5 as a function of scattered momentum $\mathbf{q}$ for initial states an the nodal $\mathbf{k}_{\text {nod }}$ and antinodal $\mathbf{k}_{\text {antinod }}$ points, accordingly, since in these directions bosonic renormalization of the electronic band (so-called «kink») was observed. Note, that the matrix element of the apex breathing mode does not depend on $\mathbf{k}$ and $\mathbf{q}$. For a nodal fermion initial state, the coupling is largest to the breathing mode for $\mathbf{q}=(\pi, 0)$ and $\mathbf{q}=(\pi, \pi)$ (see Fig. 5, $a$ ), and for the buckling mode with small value of $\mathbf{q}$ (Fig. $5, b$ ), because such phonons connect electrons with initial states $\mathbf{k} \sim \mathbf{k}_{F}$ to the final states on the Fermi surface. For an antinodal fermion initial state, the breathing coupling is weak (Fig. 5,c), while the buckling interaction has a maximal value for small $\mathbf{q}$ (Fig. $5, d$ ).

To analyze a possible kink formation [35] we take into account the momentum dependence of the coupling and consider conservation of energy and momentum as- 
suming that the electron energy in the superconducting phase is given by the BCS formula $E(k)= \pm \sqrt{\varepsilon_{k}^{2}+\Delta_{k}^{2}}$ with order parameter having $d$-wave symmetry. At the nodal point $\mathbf{k}_{\text {nod }}=((1-\delta) \pi / 2,(1-\delta) \pi / 2), \quad \delta \leq 0.1$, the breathing mode has maximum coupling to the scattering momentum $\mathbf{q}_{1}=(\pi, \pi)$ and $\mathbf{q}_{2}=(\pi, 0)$. At the same time we have $E\left(\mathbf{k}_{\text {nod }}-\mathbf{q}_{1}\right) \approx \Delta\left(\mathbf{k}_{\text {nod }}-\mathbf{q}_{1}\right)=0$, and thus a kink energy is equal to $\varepsilon\left(\mathbf{k}_{\text {nod }}\right)=\left|E\left(\mathbf{k}_{\text {nod }}-\mathbf{q}\right)-\omega_{q_{1}}\right|=70 \mathrm{meV}$. This energy corresponds to the breathing mode frequency at $\mathbf{q}_{1}=(\pi, \pi)$. Similarly the mode with $\mathbf{q}_{2}$ also satisfies conservation of energy. In that way we find that the contribution to electron spectrum renormalization comes from coupling to a breathing mode with $\mathbf{q}_{1}=(\pi, \pi)$ and $\mathbf{q}_{2}=(0, \pi)$ at the nodal point, and from coupling to a buckling mode with small value of $\mathbf{q}$ at the antinodal point.

For constructing the theory of superconductivity it is of necessity to obtain an effective Hamiltonian where EPI is excluded in the manner of Fröhlich transformation [36]. For the low energy single-band $t-J^{*}$ model this transformation is not quite trivial. To perform it we neglect the interband excitations and apply Hubbard-I-type approximation. As a result, the effective Hamiltonian can be written in the following form

$$
\begin{gathered}
H_{\mathrm{eff}}=H_{t-J^{*}}+H_{\mathrm{el}-\mathrm{ph}-\mathrm{el}}, \\
H_{\mathrm{el}-\mathrm{ph}-\mathrm{el}}=\sum_{\mathbf{k} \mathbf{k}^{\prime} \mathbf{q} v} \sum_{m} V_{\mathbf{k k}^{\prime} \mathbf{q}}^{m m}\left(X_{\mathbf{k}+\mathbf{q}}^{m}\right)^{+}\left(X_{\mathbf{k}^{\prime}-\mathbf{q}}^{m}\right)^{+} X_{\mathbf{k}^{\prime}}^{m} X_{\mathbf{k}}^{m},
\end{gathered}
$$

$$
\begin{aligned}
& V_{\mathbf{k k}^{\prime} \mathbf{q}}^{m m}=g_{m m}^{(v)}(\mathbf{k}, \mathbf{q}) g_{m m}^{(v)}(\mathbf{k},-\mathbf{q})_{\Theta}{ }_{q, v} \times \\
& \times\left[\left(t_{m}(\mathbf{k})-t_{m}(\mathbf{k}+\mathbf{q})\right)^{2} F_{m}^{2}-\omega_{\mathbf{q}, v}^{2}\right]^{-1},
\end{aligned}
$$

where $H_{t-J^{*}}$ is low energy single-band $t-J^{*}$ model, quasiparticle index $m$ is different for cuprates with hole or electron doping type, $t_{m}(\mathbf{k})$ is the dispersion of the free electrons. Here $V_{\mathbf{k k}^{\prime} \mathrm{q}}^{m m}$ is the effective electron-electron interaction, which depends on the occupation factors $F_{m}$ and hence on the doping concentration, temperature, and magnetic field, in contrast to the theory of weakly correlated electrons. The construction of the theory of superconductivity with both magnetic and phonon mechanisms of pairing in the regime of SEC is still not finished; nevertheless we hope such a theory may give answers to the main unsolved problems concerning the unusual aspects of the normal and superconducting properties of the single layer cuprates. Concerning the effect of interlayer coupling and increasing $T_{c}$ over $100 \mathrm{~K}$ in $\mathrm{Y}^{-}, \mathrm{Bi}^{-}, \mathrm{Tl}-$, and Hg-based cuprates, these are another set of problems we have not considered here.

\section{Conclusion}

The GTB method and its ab initio modification described in this paper give a correct description of the undoped and weakly doped cuprates both with hole and electron doping. It also allows one to construct a realistic effective Hamiltonian to study the low-energy physics of strongly correlated electrons interacting with spin fluctuations and phonons. A lot of work still has be done to understand the normal and superconducting states in cuprates, and we believe our approach provides a solid background for future progress in this field.

This work has been supported by RFBR grant 03-02-16124 and Russian Academy of Science Program «Quantum Macrophysics». One of the authors (E.I.S.) acknowledges support from Dynasty Foundation and ICFPM, and «Russian Science Support Foundation» .

1. J.G. Bednorz and K.A. Muller, Z. Phys. B64, 189 (1986).

2. V.L. Ginzburg and E.G. Maksimov, Superconductivity: Physics, Chemistry, and Technology 5, 1543 (1992), (in Russian).

3. E. Dagotto, Rev. Mod. Phys. 66, 763 (1994).

4. V.M. Loktev, Low Temp. Phys. 22, 1 (1996).

5. S.G. Ovchinnikov, Physics - Uspekhi 40, 993 (1997).

6. E.G. Maksimov, Physics - Uspekhi 43, 965 (2000).

7. S.G. Ovchinnikov and I.S. Sandalov, Physica C161, 607 (1989).

8. V.A. Gavrichkov, S.G. Ovchinnikov, A.A. Borisov, and E.G. Goryachev, JETP 91, 369 (2000).

9. S.G. Ovchinnikov and V.V. Val'kov, Hubbard Operators in the Theory of Strongly Correlated Electrons, Imperial College Press, London-Singapore (2004).

10. P. Fulde, Electron Correlations in Molecules and Solids, Springer-Verlag, Berlin (1991).

11. S.G. Ovchinnikov, Acta Phys. Polon. B34, 431 (2003).

12. H. Lehmann, Nuovo Cim. 11, 342 (1954).

13. E.M. Lifshitz and L.P. Pitaevskii, Course of Theoretical Physics,: Statistical Physics, Nauka, Moskow (1978), v. 5; Pergamon, New York (1980), part 2.

14. J.C. Hubbard, Proc. Roy. Soc. A285, 542 (1965).

15. R.O. Zaitsev, JETP 43, 574 (1976).

16. Yu.A. Izyumov, M.I. Katsnelson, and Yu.N. Skryabin, Magnetism of Collectivized Electrons, Fizmatgiz, Moskow (1994).

17. V.G. Bar'yakhtar, V.N. Krivoruchko, and D.A. Yablonskii, Green's Functions in Magnetism Theory, Naukova Dumka, Kiev (1984) (in Russian).

18. Ya.B. Gaididei and V.M. Loktev, Phys. Status Solidi B147, 307 (1988). 
19. A.A. Borisov, V.A. Gavrichkov, and S.G. Ovchinnikov, JETP 97, 773 (2003).

20. M.M. Korshunov, V.A. Gavrichkov, S.G. Ovchinnikov, Z.V. Pchelkina, I.A. Nekrasov, M.A. Korotin, and V.I. Anisimov, JETP 99, 559 (2004).

21. V.J. Emery, Phys. Rev. Lett. 58, 2794 (1987).

22. C.M. Varma, S. Schmitt-Rink, and E. Abrahams, Solid State Commun. 62, 681 (1987).

23. C. Janowitz, U. Seidel, R.-S.T. Under, A. Krapf, R. Manzke, V.A. Gavrichkov, and S.G. Ovchinnikov, JETP Lett. 80, 692 (2004).

24. S.G. Ovchinnikov, A.A. Borisov, V.A. Gavrichkov, and M.M. Korshunov, J. Phys.: Condens. Matter 16, L93 (2004).

25. M.M. Korshunov, V.A. Gavrichkov, S.G. Ovchinnikov, D. Manske, and I. Eremin, Physica C402, 365 (2004).

26. N. Harima, J. Matsuno, A. Fujimori, Y. Onose, Y. Taguchi, and Y. Tokura, Phys. Rev. B64, 220507 (2001).

27. M.M. Korshunov and S.G. Ovchinnikov, Phys. Solid State 43, 416 (2001).
28. M.M. Korshunov, S.G. Ovchinnikov, and A.V. Sherman, JETP Lett. 80, 39 (2004).

29. V.V. Val'kov, T.A. Val'kova, D.M. Dzebisashvili, and S.G. Ovchinnikov, JETP Lett. 75, 378 (2002).

30. V.A. Gavrichkov, M.M. Korshunov, and S.G. Ovchinnikov, Int. J. Mod. Phys. B19, 247 (2005).

31. V.Yu. Yushankhay, N.M. Plakida, and P. Kalinay, Physica C174, 401 (1991).

32. Yu. A. Izyumov, Usp. Fiz. Nauk 167, 465 (1997).

33. S.G. Ovchinnikov and E.I. Shneyder, JETP 101, 844 (2005).

34. T.S. Nunner, J. Schmailian, and K.N. Bennemann, Phys. Rev. B59, 8859 (1999).

35. A. Lanzara, P.V. Bogdanov, X.J. Zhou, S.A. Kellar, D.L. Feng, E.D. Lu, T. Yoshida, H. Eisaki, A. Fujimori, K. Kishio, J.-I. Shimoyama, T. Noda, S. Uchida, Z. Hussain, and Z.X. Shen, Nature 412, 510 (2001).

36. H. Fröhlich, Phys. Rev. 79, 845 (1950) 